\title{
Low frequency heating and flow driven by the dynamic ergodic divertor in tokamaks
}

\author{
A.G. Elfimov ${ }^{1}$, D.W. Faulconer ${ }^{2, \text { a }}$, K.H. Finken ${ }^{3, a}$, \\ R.M.O. Galvão ${ }^{1}$, A.A. Ivanov $^{4}$, R. Koch ${ }^{2, a}$, S.Yu. Medvedev ${ }^{4}$ and \\ R. Weynants ${ }^{2, a}$ \\ ${ }^{1}$ Physics Institute, University of São Paulo, 05508-900, São Paulo, Brazil \\ ${ }^{2}$ Laboratory for Plasma Physics, Koninklijke Militaire School-Ecole Royale Militaire, \\ Brussels, Belgium \\ ${ }^{3}$ Institut für Plasmafysik, Forschungszentrum Jülich GmbH, Jülich, Germany \\ ${ }^{4}$ Keldysh Institute, Russian Academy of Sciences, Moscow, Russia
}

Received 19 November 2003, accepted for publication 28 April 2004

Published 28 May 2004

Online at stacks.iop.org/NF/44/S83

doi:10.1088/0029-5515/44/6/S09

\begin{abstract}
The profile and dissipation of the field excited by dynamic ergodic divertor (DED) coils in tokamak plasmas are calculated, and an estimate is made of the poloidal/toroidal velocities driven by this field. The coils are idealized as an inboard sheet current composed of a toroidal sequence of helical line current segments expanded in Fourier series with poloidal/toroidal mode numbers $M / N$, and mode amplitudes depending on feeding. Numerical calculations with cylindrical and toroidal codes show maxima of field dissipation due to Alfvén wave mode conversion effect taking place at the rational magnetic surfaces where $q=M / N$. The effects of toroidicity and ion collisions in the dielectric tensor in the upper DED frequency range described $(f=5-10 \mathrm{kHz})$ are found to be very important in absorption calculations. At the $q=3$ resonant magnetic surface typical for DED coil design, it is estimated that ponderomotive forces produced by $20 \mathrm{~kW}$ of dissipation can drive local toroidal and poloidal flows of respective orders $8 \mathrm{~km} \mathrm{~s}^{-1}$ and $10 \mathrm{~km} \mathrm{~s}^{-1}$ in the TEXTOR tokamak.
\end{abstract}

PACS numbers: 52.65.Kj, 52.65.Tt, 52.35.Bj, 52.55.Fa, 52.55.Rk

\section{Introduction}

In view of the demonstrated advantageous magnetic topology already afforded by a static ergodic divertor (see, e.g. [1]), dynamic ergodic divertor (DED) coils have been proposed as a means to favourably influence heat exhaust, edge cooling, impurity screening, plasma confinement and stability at the plasma boundary (see [2] for motivation and detailed description of DED coils for the TEXTOR tokamak). Related earlier experimental and theoretical studies on penetration of rotating low frequency (LF) fields into tokamak plasmas [3,4] continues in recent experiments on the HYBTOK-II tokamak [5]. More exclusively theoretically oriented study is an active endeavour [6-12] which includes modelling of DED coils for the Tokamak Chauffage Alfvén Brésilien (TCABR) [9]. The present work models the DED coils recently installed on TEXTOR, treating them as an antenna producing energy deposition and associated plasma flow in the upper DED frequency band $5-10 \mathrm{kHz}$, these quantities being presumably linked to the positive DED features cited above. Developing the coil current distribution in Fourier series, we analyse

a Trilateral Euregio Cluster. the penetration depth of the LF fields and their dissipation at the rational magnetic surfaces where $q=M / N$, about which the Alfvén resonance and associated strong interaction with the plasma take place $(M / N$ are poloidal/toroidal mode numbers and $q$ is the safety factor). The DED coils have a helicity such as to excite preferentially mode pairs $M, N$ having resonant surfaces near the plasma boundary in order to affect edge transport.

To analyse LF field dissipation we use cylindrical kinetic $[13,14]$ ('cylindrical' hereafter) and two-fluid toroidal [15] ('toroidal' hereafter) codes developed for the calculation of Alfvén wave excitation and dissipation in tokamaks. Besides retention of parallel electron-kinetic and finite ion Larmor radius contributions in the cylindrical code (enabling full evaluation of the ponderomotive forces driving plasma flow), and the toroidal geometry of the toroidal one, the plasma description used in both codes has been rendered identical (strongly collisional). Application of the former code for the cylindrical limit provides a finer radial mesh and spares us intervention in the latter toroidal code to adapt an embedded toroidal equilibrium calculation to this limit. The cylindrical code is used to examine the effect of finite Larmor radius on the 
structure of the fields at the resonance surface and to evaluate the ponderomotive effects underlying the production of plasma rotation; its toroidal counterpart is here reserved for study of specifically toroidal effects such as poloidal mode coupling and associated poloidal asymmetry of power deposition. Consistent with the cross sections of tokamaks of present DED interest, both codes compute fields and dissipation assuming circular magnetic surfaces (with Shafranov shift in the toroidal code). The possible relevant effects of magnetic islands on Alfvén wave resonances pointed out in [7] are not included in the present analysis. Explicitly, we assume that the width of the magnetic islands is smaller than the distance between two local Alfvén resonances and that the velocity oscillation induced by the Alfvén waves is much smaller than the poloidal phase velocity of the DED field.

To calculate ponderomotive forces that drive current and plasma flow, we use an approach based on averaging of twofluid plasma equations over magnetic surfaces developed in [16]. Using dissipation profiles calculated with the codes and expressions for ponderomotive forces taken from this reference, we make rough estimates of the poloidal and toroidal flows driven by the DED coils in the TEXTOR plasma.

This paper is organized as follows. In section 2, we briefly describe the antenna and collisional plasma models used in the two codes. Section 3 deals with plasma parameters used and specific aspects of the DED coil model chosen. Section 4 begins with a brief theoretical overview useful in understanding a subsequent presentation of the results of code calculations of the LF field distribution and dissipation over the tokamak cross section, results from the cylindrical code being followed by a study of toroidal effects. In section 5, we calculate and discuss the ponderomotive forces and plasma poloidal and toroidal flows driven by the DED coils in TEXTOR. Finally, we summarize the main results of the calculations and present our conclusions in section 6 .

\section{Codes used}

To calculate LF field excitation in the discussed plasmas we use the cylindrical and toroidal codes mentioned in the introduction $[14,15]$. The conditions for their validity, away from the rational surfaces where $k_{\|}=0$, are satisfied in a peripheral (cold plasma) region extending over approximately the outer third of the minor radius, in a frequency band that is determined by inequalities

$$
v_{\text {ii }} \leqslant \omega \ll v_{\text {ei }} \ll k_{\|} v_{T_{\mathrm{e}}} \leqslant k_{\|} c_{\mathrm{A}},
$$

where $k_{\|}=(m / r) B_{0 \theta} / B_{0}+\left(n / R_{0}\right) B_{0 \phi} / B_{0}$ is the cylindrical parallel wavenumber, $v_{T_{\mathrm{i}, \mathrm{e}}}$ is the ion or electron thermal velocity, $v_{\mathrm{ii}}, \quad v_{\mathrm{ei}}$ are ion-ion and electron-ion collision frequencies, and $c_{\mathrm{A}}=B_{0} /\left(4 \pi n_{\mathrm{i}} m_{\mathrm{i}}\right)^{1 / 2}$ the Alfvén velocity. Around the rational surfaces where $k_{\|} \approx 0$, the inequalities (1) are modified to the following collisional MHD conditions under which both cylindrical and toroidal codes are valid $\left(v_{\mathrm{ei}} \approx 6 \times 10^{5} \mathrm{~s}^{-1}\right)$ :

$$
k_{\|} v_{T_{\mathrm{i}}} \ll v_{\mathrm{ii}}, \omega_{\mathrm{e}}^{*}, \quad \quad k_{\|} v_{T_{\mathrm{e}}}<k_{\|} c_{\mathrm{A}}, \quad \omega \ll v_{\mathrm{ei}},
$$

where $\omega_{\mathrm{e}}^{*}$ is the electron drift frequency $\left(v_{T_{\mathrm{e}}} M \rho_{\mathrm{e}} / r\right) \partial\left(\ln T_{\mathrm{e}} n_{\mathrm{e}}\right) /$ $\partial r$. In figure 1 , we show the characteristic dependence on

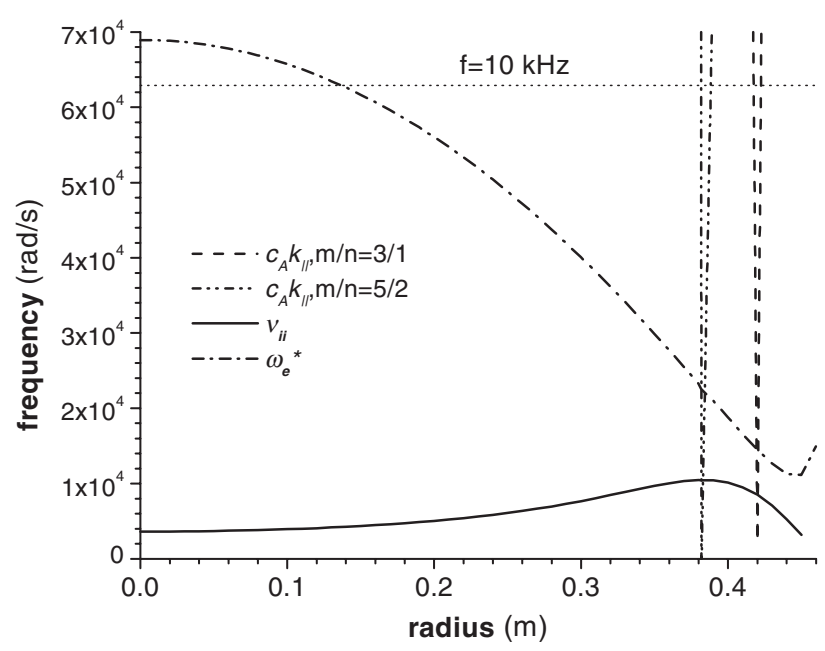

Figure 1. Plot of ion collision $v_{\mathrm{ii}}\left[\mathrm{s}^{-1}\right]$, drift $\omega_{\mathrm{e}}^{*}$, and local Alfvén $k_{\| \mid} c_{\mathrm{A}}$ frequencies over the minor radius at the poloidal angle $\pi / 2$ for the typical parameters of TEXTOR plasmas presented in section 3 , with temperatures $T_{\mathrm{ea}}=80 \mathrm{eV}$ and $T_{\mathrm{ia}}=140 \mathrm{eV}$.

radius of these TEXTOR parameters. Here we observe that in the outer region of the plasma the ion collision frequency is of the order of the electromagnetic field frequency and may be important in the evaluation of LF field dissipation.

\subsection{Cylindrical code}

The cylindrical code's plasma model is formulated in terms of the Maxwell equations and a dielectric tensor for a two-ion species magnetized plasma with circular concentric magnetic surfaces, this being a collisional adaptation of an earlier kinetic version [13]. Linking cylindrical geometry to a toroidal one by the standard replacement of $k_{z}$ by $N / R_{0}$ where $N$ and $R_{0}$ are, respectively, the toroidal mode number and major radius, the code calculates the distribution of electromagnetic fields, dissipation profiles, and antenna impedance for given generator frequency $\omega$ by summing over individual poloidal/toroidal antenna current modes $M / N$. We choose the dielectric tensor components used in the toroidal code in [15] (cold plasma approach of [17], $\omega>k_{\|} v_{T_{\mathrm{e}}}$ ):

$$
\begin{gathered}
\varepsilon_{11}=\varepsilon_{1}=1+\sum_{\alpha} \frac{\omega_{\mathrm{p} \alpha}^{2}}{\omega_{\mathrm{c} \alpha}^{2}-\left(\mathrm{i} \nu_{\mathrm{i}}+\omega\right)^{2}}, \\
\varepsilon_{33}=1-\frac{\omega_{\mathrm{pe}}^{2}}{\omega\left(\omega+\mathrm{i} \nu_{\mathrm{e}}\right)}-\frac{\omega_{\mathrm{pi}}^{2}}{\omega\left(\omega+\mathrm{i} v_{\mathrm{i}}\right)}, \\
\varepsilon_{12}=-\varepsilon_{21}=\mathrm{i}\left(\frac{\omega}{\omega_{\mathrm{ci}}} \varepsilon_{1}-\frac{c^{2}}{\omega^{2}} k_{\|} \frac{B_{0 \phi}^{2}}{r B_{0}^{2}} \frac{\mathrm{d}}{\mathrm{d} r}\left(\frac{r B_{0 \theta}}{B_{0 \phi}}\right)\right),
\end{gathered}
$$

where the set $(1,2,3)$ refer to normal, binormal, and parallel components relative to the equilibrium magnetic field $\boldsymbol{B}_{0}$. However, to incorporate a gradient contribution to the ponderomotive force, the cylindrical code $[13,14]$ includes an additional finite ion Larmor radius effect in the perpendicular components and a general kinetic description in the parallel component with the most important terms shown below (here, the subscripts $(r, b, \|)$ are introduced to distinguish directions 
in the cylindrical code from those in the toroidal one):

$$
\begin{gathered}
\varepsilon_{r r} \approx \varepsilon_{1}\left[1-\frac{\rho_{\mathrm{i}}^{2}}{4}\left(3 k_{r}^{2}+11 k_{\mathrm{b}}^{2}\right)\right], \\
\varepsilon_{b b} \approx \varepsilon_{1}\left[1-\frac{\rho_{\mathrm{i}}^{2}}{4}\left(11 k_{r}^{2}+3 k_{\mathrm{b}}^{2}\right)\right], \\
\varepsilon_{33}=1+\frac{\omega_{\mathrm{pe}}^{2}}{k_{\|}^{2} v_{T_{\mathrm{e}}}^{2}}\left[1+S_{\mathrm{e}} Z\left(S_{\mathrm{e}}\right)\right], \quad S_{\mathrm{e}}=\frac{\omega+\mathrm{i} v_{\mathrm{ei}}}{k_{\|} v_{T_{\mathrm{e}}}},
\end{gathered}
$$

where $v_{T_{\mathrm{e}}}$ is the thermal velocity, $k_{r}$ and $k_{\mathrm{b}}=(\mathrm{m} / r) B_{0 \phi} / B_{0}-$ $\left(n / R_{0}\right) B_{0 \theta} / B_{0}$ are the radial and binormal components of the wavevector, and $Z\left(S_{\mathrm{e}}\right)$ is the plasma dispersion function.

With $a$ and $r_{\mathrm{c}}$ being the plasma edge and DED coil radii, respectively, the DED antenna is idealized as an inboard array of 16 helical line current segments of constant pitch, equispaced toroidally on the surface $r=r_{\mathrm{c}}$. Each segment spans $2 \pi$ radians in toroidal angle as it runs from $\theta_{\mathrm{c}}$ radians below the inboard equatorial plane to $\theta_{c}$ above it. In terms of minor radius $r$ and poloidal and toroidal angles $\theta$ and $\phi$ this coil array has the Fourier representation

$$
\begin{aligned}
& J_{\theta, \phi}(r, \theta, \phi, t)=\Sigma_{M} J_{\theta, \phi}(M, N) \delta(r-b) \\
& \quad \times \exp [\mathrm{i}(M \theta+N \phi-\omega t)],
\end{aligned}
$$

where $\delta(r-b)$ is the Dirac $\delta$-function and the $J_{\theta, \phi}(M, N)$ depend on coil feeding ('coil setting' hereafter) [2]. A sum over $N$ is omitted here in view of coil feeding being chosen for dominant excitation of a single $N$-value in the plasma region where resonant interaction takes place with the DED field, and there being moreover no mode coupling over $N$ in the tokamak geometry. Noting that integer ' $n_{0}$ ' of [2] satisfies ' $n_{0}$ ' $=\pi /(2 \Delta \phi /$ coil $)=$ (number of DED coils $) / 4$, one has ' $n_{0}$ ' $=4$ in TEXTOR, giving for ' $m_{0}$ ' of the reference ' $m_{0}$ ' $=$ ' $n_{0}$ ' $\pi / \theta_{\mathrm{c}}=4 \pi / \theta_{\mathrm{c}}$. In correspondence with the single toroidal mode retained in equation (5) we keep only the $k=0$ contribution to the toroidal spectrum in the reference and substitute for ' $m_{0}$ ' to find in TEXTOR:

$$
\begin{gathered}
J(M, N)=\frac{8 I_{\mathrm{d}}}{r_{\mathrm{c}} \theta_{\mathrm{c}}}(-1)^{\left(M-M_{0}\right)} \frac{\sin \left(M-M_{0}\right) \theta_{\mathrm{c}}}{\left(M-M_{0}\right) \pi}, \\
\theta_{\mathrm{c}}=\frac{N \pi}{M_{0}},
\end{gathered}
$$

$M_{0}$ and $N$ being, respectively, ' $n$ ' ' $m_{0}$ ' $/ 4$ and mode number, ' $n$ ', of the reference, and $I_{\mathrm{d}}$ the amplitude of current flowing on a single coil. In standard right-handed coordinates, $(r, \theta, \phi)$, the DED coil helicity requires the use of $-\theta_{c}$ in the place of $\theta_{\mathrm{c}}$, or equivalently - ' $n_{0}$ ' in the place of ' $n_{0}$ ' in the formulae derived therein. Note that the scalar coil current density $J$ in equations (5) and (6) refers to the full component of current $\boldsymbol{J}$ flowing along the coil, with components $J_{\theta, \phi}(r, \theta, \phi, t)$ and $J_{\theta, \phi}(M, N)$ following from the $\theta, \phi$ projections which introduce the sine or cosine of the angle between the coil and cylinder-axis $(\phi)$ directions.

\subsection{Toroidal code}

A multi-fluid cold plasma model is employed in the ALTOK code [15] that accommodates two-dimensional inhomogeneity and an arbitrary cross section of tokamak magnetic surfaces (taken to be circular in the present calculation) consistently determined from equilibrium with the Shafranov shift. This toroidal code includes in the dielectric tensor the electronion collisional dissipation and electron inertia in the parallel component that is valid for analyses of LF dissipation in cold and/or collisional plasmas (see equation (3)). In contrast to the cylindrical code, the toroidal one evaluates the coil current component $J_{\theta}(M, N)$ from $J_{\phi}(M, N)$ found from equations (5) and (6) by imposing $\boldsymbol{\nabla} \cdot \boldsymbol{J}=0$ on the surface $r=r_{\mathrm{c}}$; note that the use of this condition implicitly includes surface currents additional to those used for the cylindrical code. A similar antenna model with $\boldsymbol{\nabla} \cdot \boldsymbol{J}=0$ has been used for analyses of Alfvén wave dissipation in tokamaks (see, e.g. $[13,14])$.

The toroidal code uses $356 \times 79$ radial $\times$ poloidal mesh points $(311 \times 79$ in plasma $)$ to describe the coupling over the poloidal mode number $m$ produced by plasma toroidicity; such toroidicity introducing $m$-values in the plasma lie outside the original antenna spectrum. Due to this, small letters $m$ will be used to designate plasma modes, with capital $M$ reserved for the mode spectrum of coil current.

\subsection{Notation}

In addition to the use of small $m$ to specify poloidal plasma modes as distinct from those of the antenna, $M / N$ will indicate a single antenna mode (possibly normalized) in the summand of equation (5), and the so-called 'coil setting' $\left[M_{0}, N\right]$ will refer to the full sum in equation (5), it being implicit that the individual coil phasing is chosen as in [2] so as to produce the indicated central $M_{0}$ and $N$ value.

\section{Plasma and coil parameters}

As equilibrium used in modelling the LF field excitation and ponderomotive forces induced by the DED coils in the frequency range $(5-10 \mathrm{kHz})$ in a TEXTOR plasma, we assume circular magnetic surfaces with simple fitting profiles of deuterium plasma parameters. A parabolic temperature profile with pedestal is taken: $T_{\mathrm{e}, \mathrm{i}}=T_{\mathrm{e}, \mathrm{i} 0}\left(1-(r / a)^{2}\right)^{2}+T_{\mathrm{e}, \mathrm{i}}$, where the central electron and ion temperatures are 1800 and $900 \mathrm{eV}$, while two temperature conditions are considered at the plasma boundary, $T_{\mathrm{ea}}=60 \mathrm{eV}, T_{\mathrm{ia}}=80 \mathrm{eV}$ and $T_{\text {ea }}=80 \mathrm{eV}, T_{\mathrm{ia}}=140 \mathrm{eV}$. The electron density profile is given by $n_{\mathrm{e}}=n_{0}\left[\left(1-(r / a)^{2}\right)\right]^{0.9}+n_{\mathrm{a}}$ with $n_{0}=6 \times 10^{19} \mathrm{~m}^{-3}$ and $n_{\mathrm{a}}=6 \times 10^{17} \mathrm{~m}^{-3}$ for TEXTOR. The ion density $n_{\mathrm{i}}$ is taken to satisfy the requirement of charge neutrality, $n_{\mathrm{i}}=n_{\mathrm{e}}$. For Spitzer resistivity we have the respective current profile $J_{\text {eq }}=J_{0}\left(1-r^{2} / a^{2}\right)^{3}$ and safety parameters $q_{0}=0.87$, $q(r=42 \mathrm{~cm})=3$ and $q(r=a)=3.46$. The plasma profiles used in the toroidal and cylindrical codes differ slightly because of the former code's toroidal equilibrium and Shafranov shift. In figure 2 we compare density distributions and $q$-profiles used in toroidal and cylindrical codes [6], where in the former case $n=n_{0}\left(1-\Psi^{0.7}\right)$ and $J_{\mathrm{eq}}=J_{0}\left(1-\Psi^{0.85}\right)^{1.6}$ with $\Psi$ being the poloidal magnetic flux.

The calculations have been carried out assuming a circular cross section tokamak geometry with the following tokamak parameters: minor radius $a=0.46 \mathrm{~m}$, major radius $R_{0}=1.75 \mathrm{~m}$, coil radius $r_{\mathrm{c}}=0.5325 \mathrm{~m}$, and $d=0.6 \mathrm{~m}$ is 


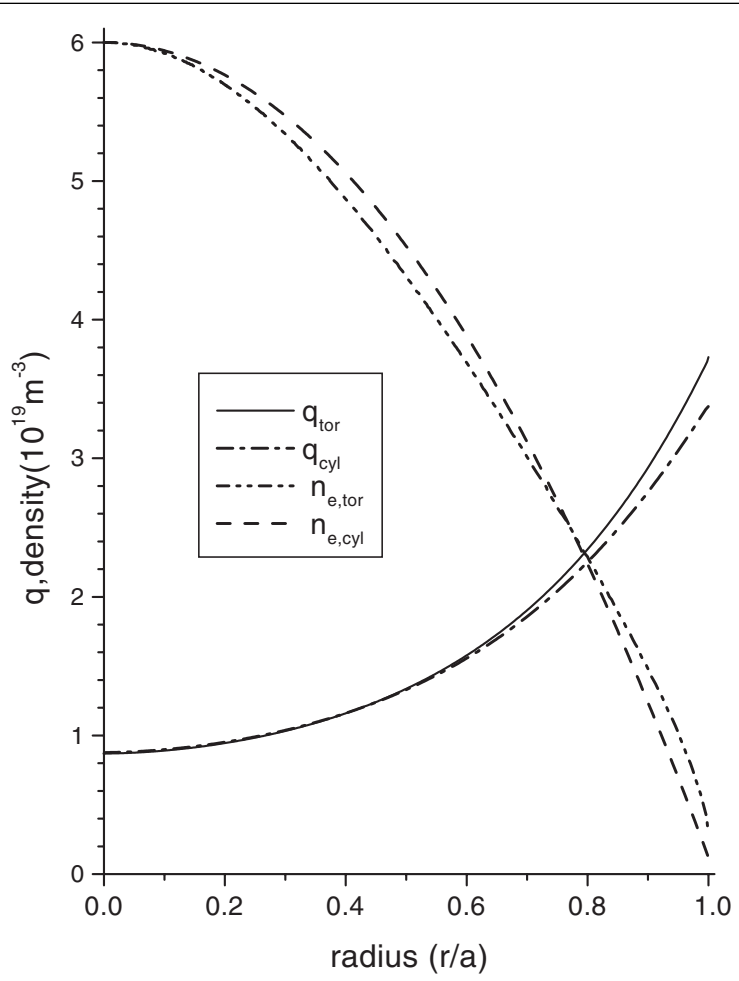

Figure 2. Density distributions and $q$-profiles used in toroidal and cylindrical codes versus normalized minor radius shown at the poloidal angle $\pi / 2$ of tokamak cross section.

the radius of the conducting wall in TEXTOR with a toroidal magnetic field $B_{0 \phi}\left(R=R_{0}\right)=2.2 \mathrm{~T}$ and Ohmic current $I_{\mathrm{p}}=370 \mathrm{kA}$. The current has the opposite direction to the toroidal magnetic field with the toroidal field circulating in the negative sense about the vertically directed symmetry axis in TEXTOR. The 16 helical DED coil segments described in section 2.1 turn about the magnetic axis in the same sense as $\boldsymbol{B}_{0}$ does, with $\theta_{\mathrm{c}}=35.6^{\circ}$ the half-width of the inboard poloidal region they occupy. Note that in standard right-handed $(r, \theta, \phi)$ coordinates $\mathrm{d} \phi / \mathrm{d} \theta<0$ in TEXTOR. To conform to the usual convention of positive $q$, we define $q$ in terms of the circuit integral of $-\mathrm{d} \phi / \mathrm{d} \theta$, giving as rational surface condition for the $M, N$ mode pair in cylindrical geometry $M=q N$.

In TEXTOR, the DED coils have been designed to excite poloidal modes having integral numbers of poloidal wavelengths, the final design admitting situations involving as lowest order pairs: three poloidal wavelengths for an $N=1$ excitation, six for $N=2$, and 12 for $N=4$. In cylindrical geometry, this dictates coil design and phasing such as to excite antenna spectra centred on the specific $M_{0} / N$ pairs $3 / 1,6 / 2,12 / 4$ [2], this entailing $\theta_{\mathrm{c}}=N \pi / M_{0}=\pi / 3$ as shown in equation (6). The above choice of $\theta_{\mathrm{c}}=35.6^{\circ}$ for the coil array implies a different ratio of $N$ to $M_{0}$, this choice having been made in anticipation of an effect of the poloidal non-uniformity of $\mathrm{d} \phi / \mathrm{d} \theta$ seen in toroidal geometry (note that [2] uses $M_{0} / N=5$ ). This effect being absent from the cylindrical geometry in which we ultimately calculate induced flow, we model an antenna with $\theta_{\mathrm{c}}=\pi / 3$. Noteworthy in equation (6) is the broad distribution of antenna sideband harmonics about the central $M_{0}$ value, this implying a correspondingly broad radial distribution of rational surfaces at which dissipation and driven flow might take place. Despite its relevance to planned DED operation, we will not examine the $[12,4]$ coil setting extensively due to numerical constraints.

\section{LF fields and dissipation excited by DED coils}

\subsection{Singular behaviour}

We briefly digress to describe certain wave characteristics useful in interpreting numerical results to be presented later. In earlier analyses of Alfvén wave heating in tokamaks that adopted a cold dielectric description as in equation (3), (but with $\varepsilon_{12}=\varepsilon_{21}=0$ in [18] through neglect of Hall current effect for $\omega \ll \omega_{\mathrm{c}}$ ), it has been shown that at a local Alfvén resonance occurring near the rational magnetic surfaces, where $q=M / N$, an incident fast wave undergoes not only enhanced collisional absorption but also mode conversion to a slow quasi-electrostatic Alfvén wave (SQAW) having dispersion $k_{r}^{2}=\left(\varepsilon_{11} \omega^{2} / c^{2}-k_{\|}^{2}\right) \varepsilon_{33} / \varepsilon_{11}$ (e.g. $\left.[13,18,19]\right)$. Such interaction takes place at paired Alfvén resonance points $r_{\mathrm{A} 1}, r_{\mathrm{A} 2}$ appearing where $\omega=\left|k_{\|} c_{\mathrm{A}}\right|$, on either side of the rational surface $r=r_{s}$ (where $k_{\|}=0$ ). The solution of the wave equation at a single $r=r_{\mathrm{A}}$ can be presented in terms of the Airy function (see [18]), which allows the evaluation of a dissipation length for the SQAW; $\Delta r_{\text {dis }} \approx 0.6 \mathrm{~cm}$ is estimated for TEXTOR and TCABR. The case of paired resonance points can be addressed by expanding the Soloviev equation [20] in Taylor series around the rational surface $r_{s}$, the resulting MHD solution taking the form outside the immediate vicinity of either resonance point [18]:

$$
\begin{array}{ll}
E_{\theta} \propto 1+\frac{r_{s}-a}{r_{s}} \ln \frac{\left|r_{\mathrm{A} 1}-r\right|}{\left|r_{\mathrm{A} 2}-r\right|} \quad \text { for } r<r_{\mathrm{A} 1}, \\
E_{\theta} \propto 1+\frac{r_{s}-a}{r_{s}} \ln \frac{\left|r_{\mathrm{A} 1}-r\right|}{\left|r_{\mathrm{A} 2}-r\right|}+\mathrm{i} \pi \quad \text { for } r_{\mathrm{A} 1}<r<r_{\mathrm{A} 2}, \\
E_{\theta} \propto 1+\frac{r_{s}-a}{r_{s}} \ln \frac{\left|r_{\mathrm{A} 1}-r\right|}{\left|r_{\mathrm{A} 2}-r\right|}+2 \mathrm{i} \pi & \text { for } r>r_{\mathrm{A} 2} .
\end{array}
$$

This equation indicates that the LF field exhibits maxima at the local Alfvén resonance points. If the dissipation length $\Delta r_{\text {dis }}$ is larger than the distance between the resonance points, the SQAW can change the field profile in equation (7) significantly. An example of this will be seen in the case of the high-mode coil setting $\left[M_{0}, N\right]=[12,4]$, the twin-peaked field profile being totally absent.

\subsection{Numerical results}

4.2.1. Cylindrical approximation. In the first step of the numerical analyses, we apply the cylindrical code [14] for one toroidal and one poloidal wavenumber with high resolution (700 radial mesh points), i.e. 12 points are distributed in $\Delta r_{\text {dis }}$ in TEXTOR. In figures 3 and 4 we show the dependence of antenna impedance on frequency, and the distribution of the $E_{r}$-component of the LF field and of the absorbed power density as a function of radius for TEXTOR parameters. Three coil settings $\left[M_{0}, N\right]=$ $[3,1],[6,2],[12,4]$ are analysed. If for a given coil setting we normalize the current spectrum in equation (6) as $J_{\text {norm }}(M, N)=J(M, N) / J\left(M_{0}, N\right)\left[\mathrm{A} \mathrm{m}^{-1}\right]$, the normed 


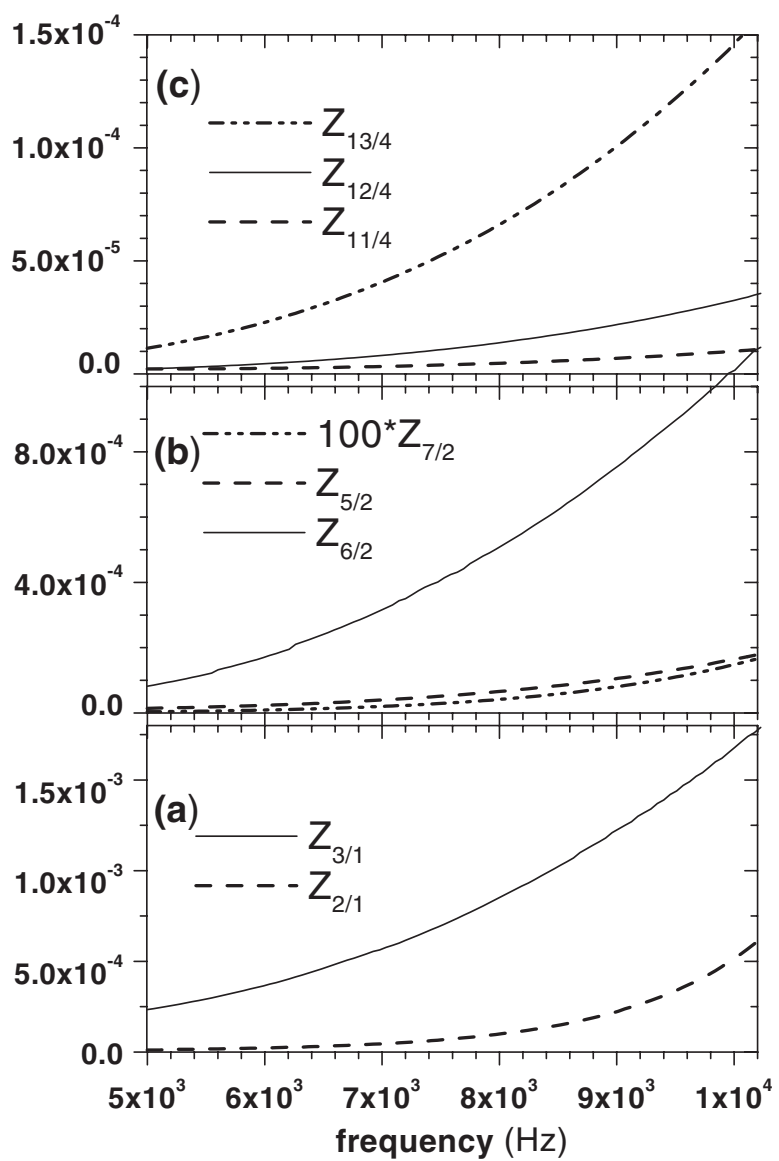

Figure 3. Plot of the antenna impedance $Z_{M / N}=W_{M / N} / I_{M / N}^{2}$ over frequency for: $(a)\left[M_{0}, N\right]=[3,1],(b)\left[M_{0}, N\right]=[6,2]$, (c) $\left[M_{0}, N\right]=[12,4]$ coil settings (cylindrical code; boundary temperatures $T_{\mathrm{ea}}=80 \mathrm{eV}, T_{\mathrm{ia}}=140 \mathrm{eV}$ in TEXTOR).

values correspond to the true dimensional current spectrum seen when the amplitude of current flowing in one coil in TEXTOR is $0.21 \mathrm{~A}$. Amplitudes of unit magnitude are taken in each numerical example $\left(J(M, N)=1\left[\mathrm{~A} \mathrm{~m}^{-1}\right]\right)$. This use of a normed current is equivalent to all first-order variables being calculated per unit current density $J(M, N)\left[\mathrm{A} \mathrm{m}^{-1}\right](\Rightarrow$ $E_{r}$ is plotted in units $\left[\left(\mathrm{V} \mathrm{m}^{-1}\right)\left(\mathrm{A} \mathrm{m}^{-1}\right)^{-1}\right]=\left[\mathrm{V} \mathrm{A}^{-1}\right], B_{\theta}$ in units $\left[\mathrm{T}\left(\mathrm{A} \mathrm{m}^{-1}\right)^{-1}\right]=\left[\mathrm{Tm} \mathrm{A}^{-1}\right], \quad P$ in units $\left[\left(\mathrm{W} \mathrm{m}^{-3}\right)\left(\mathrm{A} \mathrm{m}^{-1}\right)^{-2}\right]=\left[\mathrm{W}\left(\mathrm{A}^{2} \mathrm{~m}\right)^{-1}\right], W$ has units $\left.\left[\mathrm{W}\left(\mathrm{A} \mathrm{m}^{-1}\right)^{-2}\right]=\left[\mathrm{Wm}^{2} \mathrm{~A}^{-2}\right]\right)$. The physical variables $E_{r}, B_{\theta}, P$, and $W$ follow from their normed counterparts upon multiplication of $E_{r}$ and $B_{\theta}$ by $J(M, N)\left[\mathrm{A} \mathrm{m}^{-1}\right]$, and $P, W$ by $J(M, N)^{2}\left[\mathrm{~A}^{2} \mathrm{~m}^{-2}\right]$, the multiplications restoring conventional units to the variables. In view of the moderately flat distribution of $|J(M, N)|$ around $M_{0}$, variables found from separately normed calculations already give some crude idea of the relative importance of central and side-band $\left(M_{0} \pm 1,2, \ldots\right)$ contributions. The power absorption density in electrons and ions is calculated using the time averaged equation $P^{(e, i)}=$ $\left\langle\boldsymbol{j}^{(e, i)} \cdot \boldsymbol{E}\right\rangle_{t}$, the total absorbed power $W^{(e, i)}$ being the twodimensional integral of $P^{(e, i)}$ over the plasma minor cross section multiplied by $2 \pi R_{0}$. Variables $W$ and $P$ without superscripts indicate the sum over species of their superscripted counterparts.

Generally, the higher $\left[M_{0}, N\right]$ coil settings are associated with lower dissipation (see figure 3 ). One can likewise observe

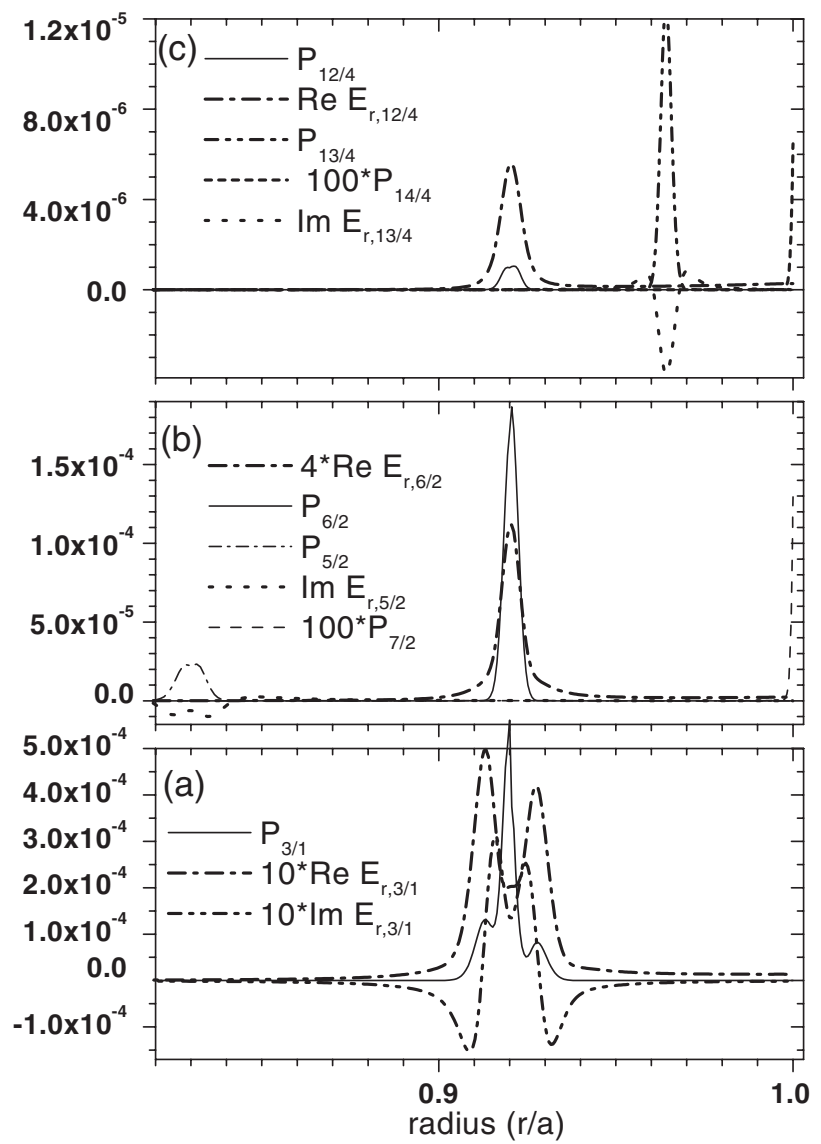

Figure 4. Plot of radial electric field $E_{r, M / N}\left[\mathrm{~V} \mathrm{~A}^{-1}\right]$ and dissipation profile $P_{M / N} \equiv r P\left[\mathrm{~W} \mathrm{~A}^{-2}\right]$ over radius excited by DED in TEXTOR with $f=10 \mathrm{kHz}$ : $(a)\left[M_{0}, N\right]=[3,1]$,

(b) $\left[M_{0}, N\right]=[6,2],(c)\left[M_{0}, N\right]=[12,4]$ coil settings (cylindrical code; same parameters as in figure 3 with current normed so that $\left.J(M, N)=1 \mathrm{~A} \mathrm{~m}^{-1}\right)$.

that the strength of LF field absorption from the central spectral amplitude, $M_{0} / N=3$, diminishes strongly with growing mode number (resonance near fixed position, $r_{s}=42 \mathrm{~cm}$ for TEXTOR). Comparing the DED sideband and main harmonic dissipation, we observe that sidebands with mode numbers not corresponding to a rational surface in the plasma only produce skin layer dissipation; this is rather small in comparison with the deposition from the other modes at their rational surfaces. The field of these sidebands is mainly concentrated in vacuum with the maximum field value occurring for the harmonic nearest to the resonance harmonic (e.g. $M=4$ for the $[3,1]$ coil setting). Large dissipation is produced by the harmonics having a resonance surface in the plasma situated close to its boundary. Upper sideband harmonics, with the resonance surface position close to the plasma surface (e.g. the $13 / 4$ sideband), have a larger dissipation than the lower ones with the resonance position further inside the plasma surface. In the case of high sideband harmonic number with local Alfvén resonance on the plasma border, for example, for the $M / N=14 / 4$ mode, the dissipation exhibits a skin layer profile. Finally, we note that in figure 4 , the $E_{r}$ field shows a merging of closely paired resonance peaks when the mode numbers of the $\left[M_{0}, N\right]$ settings grow, as was discussed in section 4.1. The twin-peaked dissipation feature, associated 

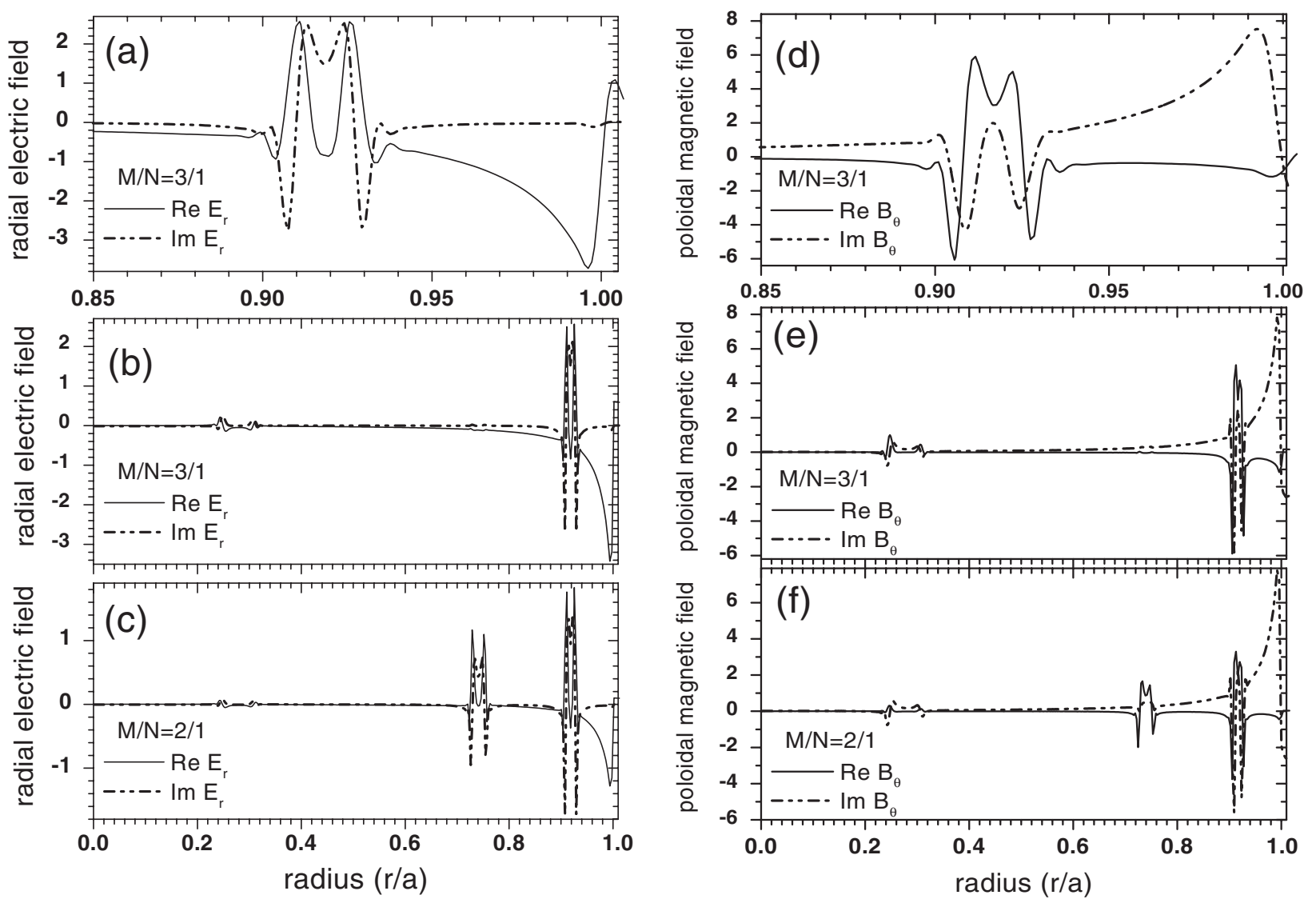

Figure 5. Plot of real and imaginary parts (- and - - - - of $E_{r, M / N}\left[10^{-5} \mathrm{~V} \mathrm{~A}^{-1}\right]$ and $B_{\theta, M / N}\left[10^{-8} \mathrm{Tm} \mathrm{A}^{-1}\right]$ field components for $[3,1]$ coil setting in TEXTOR with current normed so that $J(M, N)=1 \mathrm{~A} \mathrm{~m}^{-1}$. Plasma response for $f=10 \mathrm{kHz}$ calculated by the toroidal code with $311 \times 79$ radial $\times$ poloidal mesh points for $(a),(b),(d)$ and $(e) M / N=3 / 1$ and $(c)$ and $(f) 2 / 1$ antenna mode numbers

with the finite Larmor radius effect, and the corresponding field structure of the $3 / 1$ mode seen for $T_{\text {ea }}=80 \mathrm{eV}$ and $T_{\mathrm{ia}}=140 \mathrm{eV}$ are absent when the lower plasma boundary temperature is taken, $T_{\mathrm{ea}}=60 \mathrm{eV}$ and $T_{\mathrm{ia}}=80 \mathrm{eV}$. The latter case is used for calculations of the normed dissipation and driving force profiles displayed in figure 8 . We note that only a minor difference is found between these absorption profiles calculated with toroidal and cylindrical codes.

In figure 3 , the coil current $I_{M / N}$ is expressed through the surface current from equation (5): $I_{M / N} \approx$ $\left|\left(2 r_{\mathrm{c}} / M\right) J(M, N)\right|=\left|\left(2 r_{\mathrm{c}} / M\right) J_{\text {norm }}(M, N)\left(I_{\mathrm{d}} / 0.21\right)\right|[A]$, allowing us to calculate $W_{M / N}$ from the plotted $Z_{M / N}$ in the non-normed case $J(M, N) \neq 1\left[\mathrm{~A} \mathrm{~m}^{-1}\right]$, as done in section 5 .

4.2.2. Effect of toroidicity. To study the toroidicity effects, we use the toroidal code for analyses of the $[3,1]$ coil setting in TEXTOR. In figure 5 we show the distribution of radial and poloidal LF fields as a function of the normalized minor radius $r / a$ from the magnetic axis in the outboard equatorial plane for the $2 / 1$ and $3 / 1$ antenna modes.

In figures $5(a)$ and $(b)$, the distribution of the $E_{r}$ component around the $q=3$ resonance surface in the toroidal code calculations for $[3,1]$ coil settings appears very similar to the cylindrical code calculations in figure $4(a)$. We again observe two maxima of the $E_{r}$ component at the local Alfvén resonance points, but some difference in the field behaviour

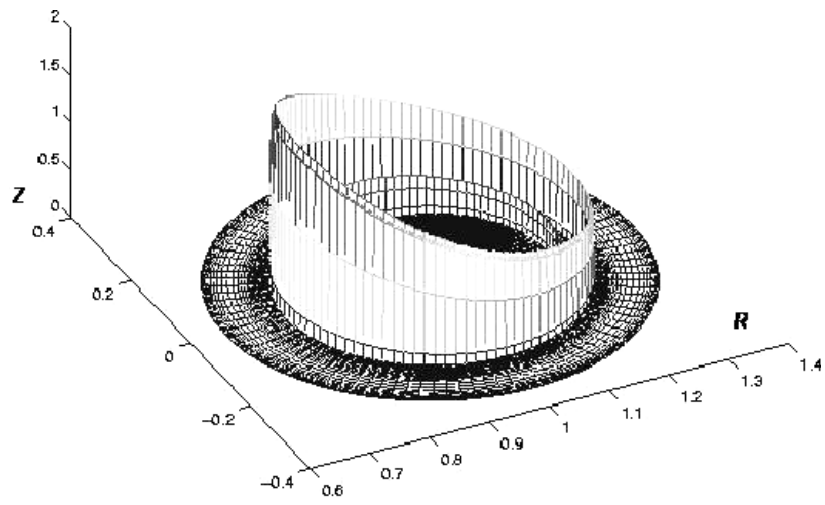

Figure 6. Plot of the LF dissipation profile $P_{2 / 1}\left[10^{-4} \mathrm{~W} \mathrm{~A}^{-2}\right]$ over the TEXTOR tokamak cross section with current normed so that $J(2,1)=1 \mathrm{~A} \mathrm{~m}^{-1}$ with $f=10 \mathrm{kHz}$ (the same case as in figure $5(c)$ and $(f)$; lengths normed to $\left.R_{0}\right)$.

appears at the rational surface due to the finite Larmor radius effect taken into account in the cylindrical code.

The dissipation profiles calculated by both codes appear very similar at the rational surfaces for the resonant poloidal modes. The toroidal code calculations of the LF field in figures $5(c)$ and $(f)$ and the absorption in figure 6 induced by the $2 / 1$ mode with $[3,1]$ coil settings show large field and strong dissipation at the $q=3$ rational surface. Of course, the 


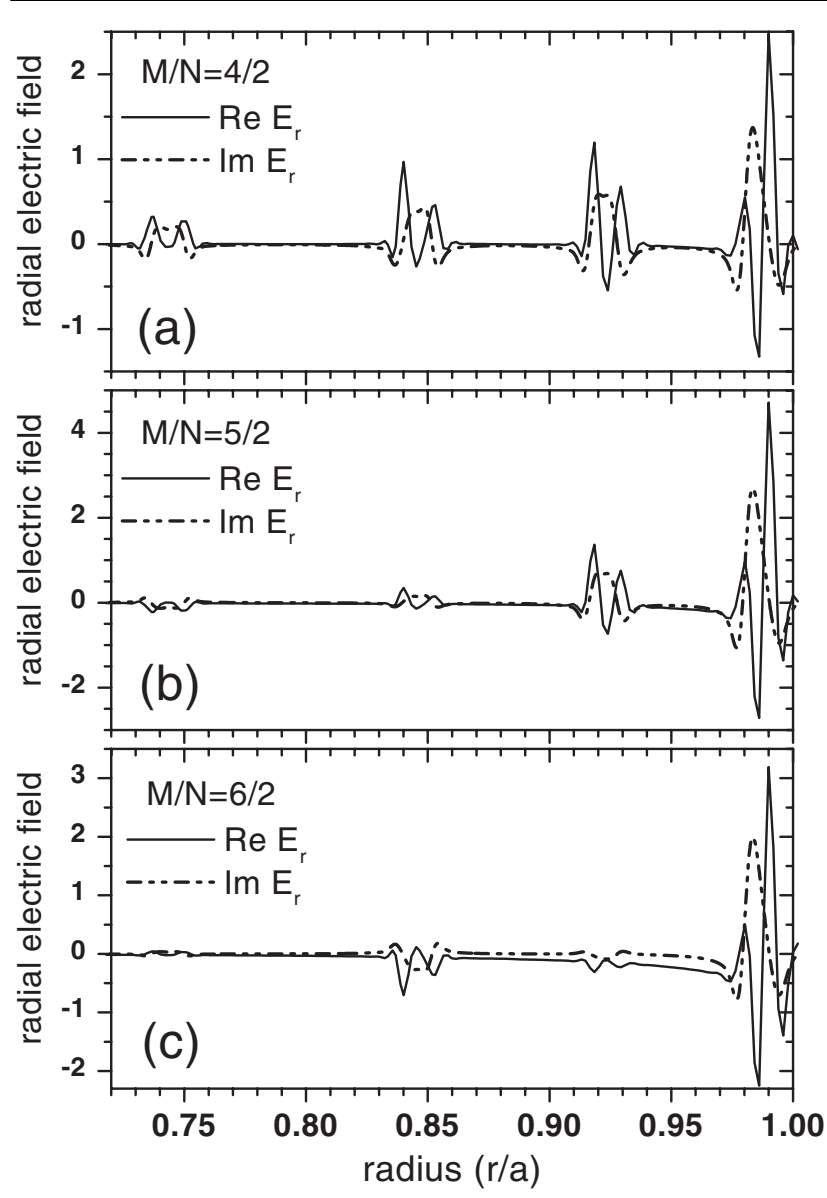

Figure 7. Plot over normed radius of real and imaginary parts of the radial electric field, $E_{r}\left[10^{-5} \mathrm{~V} \mathrm{~A}^{-1}\right]$, with current normed so that $J(M, N)=1 \mathrm{~A} \mathrm{~m}^{-1}:$ (a) $M / N=6 / 2,(b) M / N=5 / 2$, (c) $M / N=4 / 2 ;(f=10 \mathrm{kHz})$.

resonant field and dissipation at the $q=3$ surface are absent for the $2 / 1$ antenna current in the cylindrical calculations, this type of LF field excitation and absorption being produced by the coupling of poloidal modes due to plasma toroidicity. Note that very similar distributions of LF fields and dissipation are found in calculations for TCABR plasmas [9].

Turning to the $[6,2]$ scenario, $311 \times 79$ radial $\times$ poloidal mesh points in the plasma ( 45 radial points in vacuum) are used to calculate the radial electric field distribution excited by $6 / 2,5 / 2$, and $4 / 2$ antenna modes shown in figure 7 . Taking $J(M, N)=1 \mathrm{~A} \mathrm{~m}^{-1}$ and $f=10 \mathrm{kHz}$ the following respective values of normalized LF field dissipation result: $W_{\text {norm }}=1.04 \times 10^{-3}, 1.99 \times 10^{-3}, 0.726 \times 10^{-3}$. Here, we can observe that for the $[6,2]$ coil setting, all main modes are largely dissipated (or reflected) at the $q=7 / 2$ rational surface at the position $r=0.99 a$, producing a small effect at the $q=3$ rational surface.

Finally, we calculate the antenna impedance (wave dissipation divided by the square of antenna current) for $T_{\mathrm{ea}}=$ $60 \mathrm{eV}$ and $T_{\mathrm{ia}}=80 \mathrm{eV}$ with the toroidal code and compare the results with those of the cylindrical code for the central $(3 / 1)$ coil current component of the $[3,1]$ coil setting; $356 \times 79$ meshpoints are used in the former code. Both codes show similar results, allowing us to use the cylindrical code in estimating the plasma flow driven by the DED.

\section{Ponderomotive forces and rotation induced by DED coils in tokamaks}

To analyse ponderomotive forces we use the approach developed in [16] taking into account collisions and the finite Larmor radius effect [21]. The force densities acting on each plasma species $(\alpha=e, i)$ are derived in cylindrical geometry using a fluid model where they are presented as a sum of contributions stemming from fluid dynamic, electromagnetic, and viscous stresses:

$$
\begin{gathered}
F_{\mathrm{FD}, \theta, \phi}^{(\alpha)}=-\nabla\left\langle m_{\alpha} n_{\alpha} \tilde{\boldsymbol{V}}^{(\alpha)} \tilde{V}_{\theta, \phi}^{(\alpha)}\right\rangle, \\
F_{\mathrm{EM}, \theta, \phi}^{(\alpha)}=\left\langle e_{\alpha} \tilde{n}_{\alpha} \tilde{\boldsymbol{E}}+\frac{\tilde{\boldsymbol{j}}_{\alpha} \times \tilde{\boldsymbol{B}}}{c}\right\rangle_{\theta, \phi}, \\
F_{\mathrm{V}, \theta, \phi}^{(\alpha)}=-\left\langle\nabla_{s} \pi_{\mathrm{s}, \theta, \phi}^{(\alpha)}\right\rangle,
\end{gathered}
$$

where oscillatory quantities are indicated by a tilde superscript and the force densities are averaged over time and poloidal and toroidal coordinates. The model describes a magnetized plasma where the Larmor radii are much smaller than the radial scale length of the equilibrium quantities and of the LF field. Whereas the perpendicular components of the dielectric tensor are expanded to second order in ion Larmor radius, the ponderomotive contribution to $\boldsymbol{F}_{\mathrm{V}}^{(\alpha)}$ from gyroviscosity [21] is neglected. Combining the electromagnetic and fluid stresses as in [16], the result can be split into a momentum transfer contribution and one that depends explicitly on gradients of oscillating currents and fields,

$$
\begin{aligned}
&\left\langle\tilde{F}_{\theta}^{(\alpha)}\right\rangle \equiv F_{\theta, \mathrm{P}}^{(\alpha)}+F_{\theta, \partial}^{(\alpha)}=\frac{m}{r \omega} P^{(\alpha)} \\
&+ \operatorname{Re}\left\{\frac{1}{8 \pi r^{2}} \frac{\partial}{\partial r}\left[r^{2} \sum_{s=1}^{3} \varepsilon_{r s}^{(\alpha)} \hat{E}_{s}\left(\hat{E}_{\theta}^{*}-\frac{4 \pi \mathrm{i} \omega}{\omega_{\mathrm{p} \alpha}^{2}} \hat{j}_{\theta}^{(\alpha) *}\right)\right]\right\}, \\
&\left\langle\tilde{F}_{\phi}^{(\alpha)}\right\rangle \equiv F_{\phi, P}^{(\alpha)}+F_{\phi, \partial}^{(\alpha)}=\frac{N}{R_{0} \omega} P^{(\alpha)} \\
&+\operatorname{Re}\left\{\frac{1}{8 \pi r} \frac{\partial}{\partial r}\left[r \sum_{s=1}^{3} \varepsilon_{r s}^{(\alpha)} \hat{E}_{s}\left(\hat{E}_{\phi}^{*}-\frac{4 \pi \mathrm{i} \omega}{\omega_{\mathrm{p} \alpha}^{2}} \hat{j}_{\phi}^{(\alpha) *}\right)\right]\right\},
\end{aligned}
$$

where $\tilde{\boldsymbol{E}}=\hat{\boldsymbol{E}}(r, m, N) \exp [\mathrm{i}(m \theta+N \phi-\omega t)], k_{z}=N / R_{0}$, and the symbols $(s=1,2,3)$ are used for the tensor indices indicating radial, binormal, and parallel components. Here we note that the gradient force is much smaller than the momentum transfer force produced by the travelling LF field at $10 \mathrm{kHz}$ frequency. The former force is thus neglected, with the latter one serving as sole DED-induced driver of plasma rotation. Summing over species, the poloidal and toroidal components $F_{\theta P}=F_{\phi P}\left(R_{0} m / r_{s} N\right)=P m / r_{s} \omega$ of this force can be calculated using the value of LF field absorption $P$ at the rational surface found in the numerical calculations. Here, we ignore any driving effect in the skin layer (order of ion Larmor radius $\rho_{\mathrm{i}}$ ) because of large surface friction from the limiter and scrape-off layer. We assume a dissipated LF power in the plasma of about $W \approx 20 \mathrm{~kW}$, which from figure 3 can be seen to be produced by a current $I_{\mathrm{d}}$ in each coil of the order of $2 \mathrm{kA}$ amplitude for the $[3,1]$ coil setting in TEXTOR (cylindrical code, $f=10 \mathrm{kHz}$ ). This power is deposited by the $m=3$ harmonic at the rational surface 


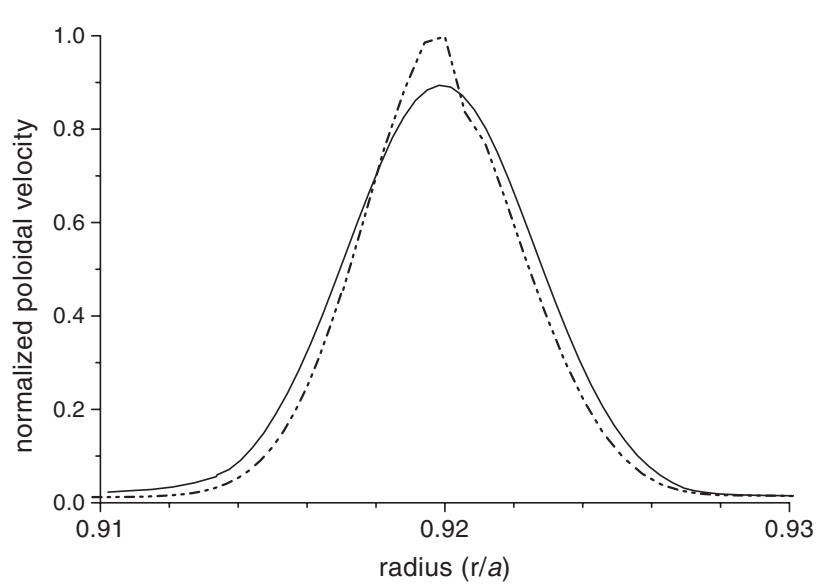

Figure 8. Plot of the radial profile of normalized DED-induced poloidal velocity evaluated in TEXTOR without $(-\cdots-)$ and with (-) Braginskii transverse viscosity $([3,1]$ coil setting at frequency $f=10 \mathrm{kHz}$; cylindrical code; case (a)).

$q_{r}=3$ in the volume $V_{r} \approx 4 \pi^{2} \Delta_{r} r_{s} R_{0}$, where $\Delta_{r}$ is the half-width of the power absorption profile $P(r)$. Since the normed 'velocity change without transverse viscosity' plotted in figure 8 is computed in fixed proportion to $F_{\theta \mathrm{P}}$, hence to $P(r)$, all three quantities have the same normed profile. The $P(r)$ half-width $\Delta_{r}$ can thus be read from this figure with appropriate multiplication of the non-dimensional abscissa by the plasma radius $a$. The average power density follows as $P \approx W / V_{r} \approx 1.4 \times 10^{5} \mathrm{~W} \mathrm{~m}^{-3}$ for the assumed $[3,1]$ coil setting. Finally, note that the single $m=3$ harmonic under discussion could contain appreciable contributions from several antenna $M$-harmonics due to poloidal coupling, yet to be included by using the toroidal code.

\subsection{Poloidal velocity}

Balancing the poloidal component of the momentum driving force just calculated with poloidal viscosity in the plateau regime [22] $\left(v_{\mathrm{ii}}<v_{T_{\mathrm{i}}} / q R_{0}\right)$ including charge exchange friction [23], we have a velocity diffusion equation in terms of the variable $x=r-r_{s} \ll r_{s}\left(r_{s}=\right.$ minor radius of singular rational surface):

$$
\begin{aligned}
& \left\langle\tilde{F}_{\theta}^{(i)}\right\rangle=F_{\text {neo }}+\frac{\partial \pi_{\theta r}^{(i)}}{\partial x}+F_{\theta, \mathrm{cx}} \Rightarrow \frac{\partial}{\partial x}\left(\eta_{1} \frac{\partial U_{\theta}^{(i)}}{\partial x}\right)-F_{\text {neo }} \\
& -F_{\theta, \mathrm{cx}}+\left\langle\tilde{F}_{\theta}^{(i)}\right\rangle=0 .
\end{aligned}
$$

Here, $\eta_{1}=0.3 m_{\mathrm{i}} n_{\mathrm{i}} \rho_{\mathrm{i}}^{2} v_{\mathrm{ii}}$ is the Braginskii transverse viscosity coefficient, $F_{\text {neo }}=m_{\mathrm{i}} n_{\mathrm{i}} \chi_{\theta}\left(U_{\theta}-\kappa_{\text {res }} v_{T_{\mathrm{i}}}\right)$ is the neoclassical viscosity force [22], where $\chi_{\theta}=\pi^{1 / 2} q v_{T_{\mathrm{i}}} /\left(2 R_{0}\right)$ and $\kappa_{\mathrm{res}} v_{T_{\mathrm{i}}}$ is the residual plasma rotation induced by the temperature gradient, and $F_{\theta, \mathrm{cx}}=m_{\mathrm{i}} n_{\mathrm{i}} n_{n}\langle\sigma v\rangle_{\mathrm{cx}} U_{\theta}$ is the charge exchange frictional force with $\langle\sigma v\rangle_{\mathrm{cx}}$ the charge exchange cross section (see [24]). In order to isolate the contribution to $U_{\theta}$ due to the DED, $\Delta U_{\theta}$, we substitute $U_{\theta}=U_{\theta}^{(0)}+\Delta U_{\theta}$ in all terms in equation (10). Using the measured characteristic parameters of the TEXTOR edge plasma (published in [2527]) at the rational surface $q_{r}=3\left(T_{\mathrm{e} r}=60 \mathrm{eV}\right.$ and $T_{\mathrm{i} r}=80 \mathrm{eV}$ for the line density $n_{\mathrm{e} r}=1.1 \times 10^{19} \mathrm{~m}^{-3}$ (a) and $T_{\mathrm{e} r}=80 \mathrm{eV}, T_{\mathrm{i} r}=140 \mathrm{eV}$ for $\left.n_{\mathrm{e} r}=0.73 \times 10^{19} \mathrm{~m}^{-3}(\mathrm{~b})\right)$ we have $\chi_{\theta} \approx 1.0-1.3 \times 10^{5} \mathrm{~s}^{-1}$. In these edge plasmas, the e-folding length of neutrals $v_{T_{n}} / n_{\mathrm{i}} /\left(3\langle\sigma v\rangle_{\mathrm{cx}}\langle\sigma v\rangle_{\mathrm{eii}}\right)^{1 / 2}$ (where $\langle\sigma v\rangle_{\text {eii }}$ is the ionization rate coefficient) is estimated to be larger than $10 \mathrm{~cm}$, which overlaps the region of the rational surface where $q=3$. Taking the neutral density at the limiter as $n_{\mathrm{na}}=0.9 \times 10^{17} \mathrm{~m}^{-3}$ [26], we find the characteristic charge exchange frequency $v_{\mathrm{cx}}=n_{n}\langle\sigma v\rangle_{\mathrm{cx}} \approx$ $0.54 \times 10^{4} \mathrm{~s}^{-1}$ at the $q=3$ rational surface. Comparing that value with $\chi_{\theta} \approx 1.0 \times 10^{5} \mathrm{~s}^{-1}$ we conclude that the poloidal rotation damping is mainly caused by the neoclassical viscosity. Using $20 \mathrm{~kW}$ absorbed power, we can produce the poloidal velocity variation, $\Delta U_{\theta} \approx(0.8-1) \times 10^{4} \mathrm{~m} \mathrm{~s}^{-1}$ for TEXTOR parameter sets (a) and (b) mentioned above, respectively. The diffusion of the poloidal velocity produced by the Braginskii transverse viscosity in equation (10) is very small (for $Z_{\text {eff }}=1.4, v_{\text {ii }}=1.7 \times 10^{4} \mathrm{~s}^{-1}$ (case (a)), the normalized diffusion coefficient $r_{s}^{-2} \eta_{1} / m_{\mathrm{i}} n_{\mathrm{i}}$ is about $1 \times 10^{-7}$ ). In figure 8 , we show the results of calculations of the velocity profile taking into account the effect of this term.

Note that for the $[6,2]$ coil setting, the same variation in plasma poloidal velocity can be achieved in TEXTOR with the current amplitude in each DED coil of the same order as the $2 \mathrm{kA}$ found for the $[3,1]$ coil setting, though this variation is foreseen at the $q=7 / 2$ rational surface at the position $r \approx 0.99 a$.

\subsection{Toroidal velocity}

Balancing the toroidal component of momentum transfer force with toroidal and ripple viscosity in the regime $\left(v_{\mathrm{ii}}<\right.$ $N_{r} v_{T_{\mathrm{i}}} / R_{0}$ ) including charge exchange friction [23,28], we have the velocity diffusion equation,

$$
\begin{gathered}
\left\langle\tilde{F}_{\phi}^{(i)}\right\rangle=F_{\text {rip }}+\frac{\partial \pi_{\phi r}^{(i)}}{\partial x}+F_{\phi, \mathrm{cx}} \Rightarrow \frac{\partial}{\partial x}\left(\eta_{2} \frac{\partial U_{\phi}^{(i)}}{\partial x}\right)-F_{\text {rip }} \\
-F_{\phi, \mathrm{cx}}+\left\langle\tilde{F}_{\phi}^{(i)}\right\rangle=0 .
\end{gathered}
$$

Here, $\eta_{2}=1.2 m_{\mathrm{i}} n_{\mathrm{i}} \rho_{\mathrm{i}}^{2} v_{\mathrm{ii}}$ is the second Braginskii transverse viscosity coefficient, $F_{\text {rip }}=m_{\mathrm{i}} n_{\mathrm{i}} \chi_{\phi} U_{\phi}$ is the ripple friction [29] where $\chi_{\phi}=1.25\left(N_{r} \delta^{2} v_{T_{\mathrm{i}}} / R_{0}\right), N_{r}$ is the number of ripples and $\delta$ is ripple modulation of the toroidal magnetic field, and $F_{\phi, \mathrm{cx}}=m_{\mathrm{i}} n_{\mathrm{i}} n_{n}\langle\sigma v\rangle_{\mathrm{cx}} U_{\phi}$ is the charge exchange friction force. Usually, the ripple modulation is very small in tokamaks and the toroidal friction is mainly defined by the charge exchange frictional force that is well known from neutral beam heating experiments (see, e.g. [28]). As was done for $U_{\theta}$ in equation (10), $\Delta U_{\phi}$ is introduced in equation (11).

Balancing the driving force $F_{\phi \mathrm{P}} \approx F_{\theta \mathrm{P}}\left(r_{s} N / R_{0} m\right)$ with charge exchange friction and assuming the same plasma parameters, $[3,1]$ coil setting and $(20 \mathrm{~kW})$ power absorbed at the $q=3$ surface as in the preceding calculations of poloidal flow, the maximum DED-induced toroidal plasma rotation velocity can be estimated as $\Delta U_{\phi \mathrm{m}} \approx(8-11) \times 10^{3} \mathrm{~m} \mathrm{~s}^{-1}$ in the respective cases (a) and (b).

The Braginskii classical transverse viscosity in equation (11) produces a substantial diffusion of the toroidal velocity. In figure 9 we show the toroidal velocity found with and without this viscosity for a normalized diffusion coefficient value $r_{s}^{-2} \eta_{2} / m_{\mathrm{i}} n_{\mathrm{i}} v_{\mathrm{cx}} \approx 0.8 \times 10^{-5}$ (case (a)). In contrast to the small diffusion of poloidal velocity found above, the maximum of the toroidal plasma velocity is reduced by diffusion to half its non-diffused value, which leads to a revised lower bound on our estimate of velocity variation $\Delta U_{\phi m} \approx(4-11) \times 10^{3} \mathrm{~m} \mathrm{~s}^{-1}$. 


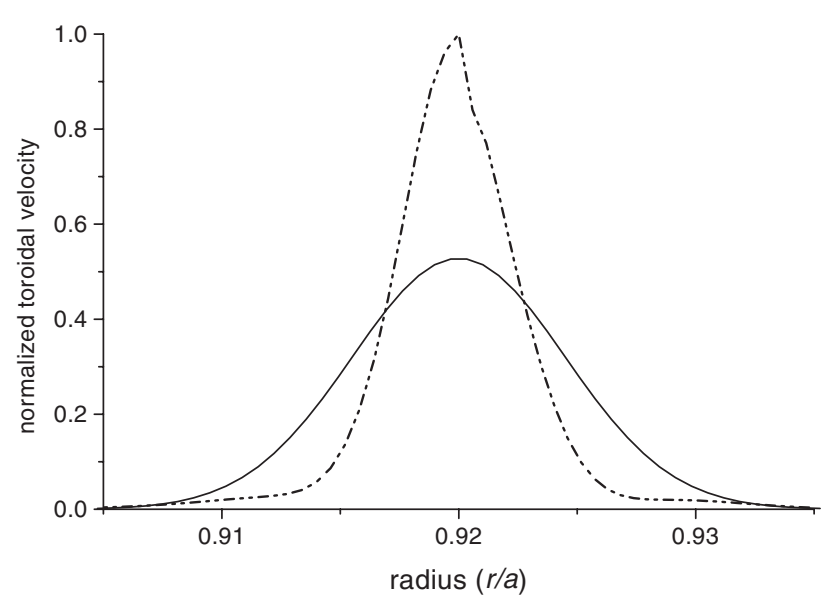

Figure 9. Plot of the radial profile of normalized DED-induced toroidal velocity evaluated in TEXTOR without $(-\cdots-)$ and with $(-)$ Braginskii parallel viscosity $([3,1]$ coil setting at frequency $f=10 \mathrm{kHz}$; cylindrical code; case (a))

\subsection{Discussion}

References [7] and [11] calculate torques exerted by the DED field on the plasma; only the former reference deals with the $[3,1]$ coil setting to which the foregoing estimations of the present section apply (see the 'resonant' case of [7]). For $f=10 \mathrm{kHz}$ it finds a 'typical' poloidal torque of $\sim 20 \mathrm{Nm}$. Under the same conditions as used for the preceding velocity evaluations ( $W=20 \mathrm{~kW}$ scales to a single-coil current amplitude $I_{\mathrm{d}}=2 \mathrm{kA}$ ) our model finds this torque value to correspond to a value $I_{\mathrm{d}} \sim 9 \mathrm{kA}$. This slightly exceeds the operating range in current of the DED at $10 \mathrm{kHz}$. In this regard one should observe that the wave dynamics of [7] differs from ours, with field resonance half-widths being an order of magnitude below the fractional centimetre values we find. This reflects the single-fluid resistive MHD description of the reference. Turning to [11], though this reference does not include a calculation of coupling to coil currents, it is worth mentioning that it finds resonance half-widths of the same length scale as ours. Neither [7] nor [11] describes the poloidal mode coupling due to toroidal geometry or the finite Larmor radius effect treated by us, though [11] includes the effect of particle drift due to a radial equilibrium field in its DED analysis.

In the above evaluation of $\Delta U_{\theta}$ and $\Delta U_{\phi}$ for the $[3,1]$ coil setting at $20 \mathrm{~kW}$ absorbed power, $F_{\phi \mathrm{P}}$ and $F_{\theta \mathrm{P}}$ are $1.3 \mathrm{~N} \mathrm{~m}^{-3}$ and $16 \mathrm{~N} \mathrm{~m}^{-3}$, respectively. An interesting comparison can be made, forces of similar magnitude having been applied in the TEXTOR plasma edge ( $44<r<46 \mathrm{~cm}$ ) by means of biasing, and the resulting velocities measured [26,27]. Modest linear extrapolation of these biasing results indicates a velocity $\Delta U_{\phi}$ of order $(4-8) \times 10^{3} \mathrm{~m} \mathrm{~s}^{-1}$ for $F_{\phi \text {, bias }}=1.3 \mathrm{~N} \mathrm{~m}^{-3}$, and $\Delta U_{\theta}$ of order $(4-8) \times 10^{4} \mathrm{~m} \mathrm{~s}^{-1}$ for $F_{\theta}$, bias $=16 \mathrm{~N} \mathrm{~m}^{-3}$, showing fair agreement with the DED-driven toroidal velocity increment calculated just above, but with the poloidal value exceeding its DED counterpart.

Note that the component of $\boldsymbol{F}_{\mathrm{P}}^{e}$ parallel to $\boldsymbol{B}_{0}$ acts on electrons to produce a small current in the resonance zone. Since this component passes through zero at the rational surface, the driven current exhibits a dipolar radial distribution about $r_{s}$. Taking $\boldsymbol{F}_{\mathrm{P}}$ as a bound for $\boldsymbol{F}_{\mathrm{P}}^{e}$ and balancing its parallel component with the electron-ion frictional force, $m_{\mathrm{e}} v_{\mathrm{ei}} n_{\mathrm{e}} \Delta V_{\mathrm{e}}$, we estimate the total current flowing in the half of this dipole lying inside $r=r_{s}$ to be $50 \mathrm{~A}$, thus implying a shift in the position of the resonance layer of only $5 \times 10^{-5} \mathrm{~m}$.

\section{Conclusions}

(1) Using the $356 \times 79$ mesh-point resolution in the toroidal code, LF fields and absorption are calculated for the cases of $[3,1]$ and $[6,2]$ DED coil settings in the TEXTOR tokamak. As a result, it has been shown that:

- strong sideband mode coupling induced by the toroidicity effect is found for all main antenna modes in both $[3,1]$ and $[6,2]$ coil settings;

- appreciable dissipation is produced at the $q=3$ rational surface by both $M=2,3$ antenna modes of the $[3,1]$ coil setting because of poloidal mode coupling;

- in the case of the $[6,2]$ coil setting, all main modes are dissipated (or reflected) at the $q=7 / 2$ rational surface producing small effect at the $q=3$ rational surface;

- to modify plasma parameters in TEXTOR at the $q=3$ rational surface the $[3,1]$ coil setting is of interest and to avoid large undesirable dissipation at the $q=4$ surface the condition $q_{\mathrm{a}}<4$ at the plasma boundary should be imposed;

- ion collisions may be important for correct calculation of the LF field dissipation.

(2) Analyses of the balance of driven and frictional forces show that:

- the momentum transfer force produced by $20 \mathrm{~kW}$ (corresponding to coil current of the order of $2 \mathrm{kA}$ for the $[3,1]$ coil setting at $10 \mathrm{kHz}$ frequency) can effectively drive toroidal and poloidal flows of respective orders 8 and $10 \mathrm{~km} \mathrm{~s}^{-1}$ at the $q=3$ rational surface in TEXTOR plasmas, and comparable flows can be driven at the $q=7 / 2$ rational surface for the $[6,2]$ coil setting with coil current amplitude of the same order;

- the poloidal flow is damped by neoclassical viscosity, the toroidal flow by the ion charge exchange frictional force.

\section{Acknowledgments}

This work is supported by the University of São Paulo and Foundation of the State of São Paulo for the Support of Research, Brazil; the Laboratory for Plasma Physics of the Koninklijke Militaire School-Ecole Royale Militaire, Belgium; and the Institut für Plasmaphysik Forschungszentrum Jülich, Germany.

\section{References}

[1] Gendrih Ph., Grossman A. and Capes H. 1996 Plasma Phys. Control. Fusion 381653

[2] Finken K.H., Abdulaev S.S., Kaleck A. and Wolf G.H. 1999 Nucl. Fusion 39637 
[3] Kobayashi M. et al 2000 Nucl. Fusion 40181

[4] Kobayashi M., Kojima H., Zhai K. and Takamura S. 2000 Phys. Plasmas 73288

[5] Takamura S., Kikuchi Y., Uesugi Y. and Kobayashi M. 2003 Nucl. Fusion 43393

[6] Faulconer D.W. and Koch R. 1997 Fusion Eng. Des. 37399

[7] Finken K.H. 1999 Nucl. Fusion 39707

[8] Finken K.H. et al 2001 Nucl. Fusion 41503

[9] Elfimov A.G. and Galvao R.M.O. 2002 29th European Conf. on Controlled Fusion and Plasma Physics (Montreux) vol 26B (ECA) P-4.108

[10] Eherer C.G., Heyn M.F., Kasilov S.V. and Kernbichler W. 2002 29th European Conf. on Controlled Fusion and Plasma Physics (Montreux) vol 26B (ECA) P-2.096

[11] Pankratov I.M., Omelchenko A.Ya. and Olshansky V.V. 2003 30th European Conf. on Controlled Fusion and Plasma Physics (St Petersburg) vol 27A (ECA) P-2.137

[12] Schillebeeckx S. and Faulconer D.W. 2002 Proc. Joint Varenna-Lausanne Workshop on Theory of Fusion Plasmas (Bologna: Societa Italiana di Fisica) p 387

[13] Ross D.W., Chen G. L. and Mahajan S.M. 1982 Phys. Fluids 25652

[14] Amarante-Segundo G., Elfimov A.G., Ross D.W., Galvao R.M.O. and Nascimento I.C. 1999 Phys. Plasmas 6 2437

[15] Galkin S.A., Ivanov A.A., Medvedev S.Yu. and Elfimov A.G. 2002 Comput. Phys. Commun. 14329
[16] Elfimov A.G., Petrzilka V. and Tataronis J.A. 1994 Phys. Plasmas 12882

[17] Ginsburg V.L. 1961 Propagation of Electromagnetic Waves in Plasmas (New York: Gordon and Breach)

[18] Elfimov A.G. 1996 Comments Plasma Phys. Control. Nucl. Fusion 17145

[19] Messiaen A.M. 1975 7th European Conf. on Controlled Fusion and Plasma Physics (Lausanne) vol 1, p 155

[20] Soloviev L.S. 1975 Reviews of Plasma Physics ed M.A. Leontovich (New York: Consultants Bureau) vol 6, p 239

[21] Elfimov A.G., Amarante-Segundo G., Galvão R.M.O. and Nascimento I.C. 2000 Phys. Rev. Lett. 841200

[22] Hirshman S.P. and Sigmar D.J. 1981 Nucl. Fusion 211079

[23] Nedospasov A.V. and Tokar M.Z. 1993 Reviews of Plasma Physics (New York: Consultants Bureau) vol 18, p 77

[24] Janev R.K. 1995 Atomic and Molecular Processes in Fusion Edge Plasmas (New York: Plenum)

[25] Huber A. et al 2002 Plasma Phys. Control. Fusion 42569

[26] Van Schoor M., Van Goubergen H. and Weynants R. 2001 J. Nucl. Mater. 290-293962

[27] Weynants R. 2001 J. Plasma Fusion Res. 43

[28] Monier-Garbet P. et al 1997 Nucl. Fusion 37403

[29] Mikhailovskii A.B. and Tsypin V.S. 1984 Sov. J. Plasma Phys. 10142 